PROCEEDINGS OF THE

AMERICAN MATHEMATICAL SOCIETY

Volume 138, Number 12, December 2010, Pages 4345-4357

S 0002-9939(2010)10436-7

Article electronically published on June 9, 2010

\title{
THE DISTRIBUTION OF ZEROS OF A CLASS OF JACOBI POLYNOMIALS
}

\author{
MARIOS CHARALAMBIDES AND GEORGE CSORDAS
}

(Communicated by Walter Van Assche)

\begin{abstract}
Polynomials whose coefficients are successive derivatives of a class of generalized Laguerre polynomials evaluated at $x=0$ are shown to be stable. These polynomials can be expressed in terms of Jacobi polynomials. The authors also prove that a related family of polynomials, depending on a parameter, possess only real and negative zeros. A special class of stability-preserving operators is also investigated.
\end{abstract}

\section{INTRODUCTION}

A real polynomial, $p(z)$, is said to be stable (or a Hurwitz polynomial) if all the zeros of $p$ lie in the open left half-plane, $\operatorname{Re} z<0$. It is frequently said that the interest in the study of stable polynomials stems from the fact that if the zeros of the eigenequation of a differential equation lie in the left half-plane, then the solution of the differential equation is stable. But in fact stability analysis plays a pivotal role in many areas of pure and applied mathematics. Here we merely cite a few topics and recent papers that have inspired our interest in stability theory and motivated the present work: the Routh-Hurwitz problem (M. Marden 19, Ch. IX]), matrix analysis (O. Holtz [15]), stability-preserving operators (T. Craven and G. Csordas 10]), differential equations and numerical analysis (D. Gottlieb [12] and D. Gottlieb and L. Lustman [13]), the Jacobi Tau class of spectral methods and eigenvalue problems (F. Waleffe [27] and M. Charalambides and F. Waleffe 4, [5]).

The characterization of stable polynomials that is most useful for our purposes is given by the Hermite-Biehler Theorem ([13, [24, p. 197]) in terms of positive pairs, defined below.

Definition 1. Two real polynomials $\Omega_{1}$ and $\Omega_{2}$ of degree $n$ and $n-1$ (or $n$ ), respectively, form a positive pair if: (a) the zeros $z_{1}, \ldots, z_{n}$ of $\Omega_{1}$ and $z_{1}^{\prime}, \ldots, z_{n-1}^{\prime}$ (or $z_{1}^{\prime}, \ldots, z_{n}^{\prime}$ ) of $\Omega_{2}$ are real, negative and distinct; (b) the zeros strictly interlace (or alternate) as follows: $z_{1}<z_{1}^{\prime}<\cdots<z_{n-1}^{\prime}<z_{n}<0$ (or $z_{1}^{\prime}<z_{1}<\cdots<z_{n}^{\prime}<$ $z_{n}<0$ ); (c) the highest coefficients of $\Omega_{1}$ and $\Omega_{2}$ are of like sign.

Theorem 2 (Hermite-Biehler Theorem). A real polynomial $p(z)=\Omega_{1}\left(z^{2}\right)+$ $z \Omega_{2}\left(z^{2}\right)$ is stable if and only if $\Omega_{1}(z)$ and $\Omega_{2}(z)$ form a positive pair.

Received by the editors June 10, 2009 and, in revised form, February 9, 2010.

2010 Mathematics Subject Classification. Primary 33C47, 26C10; Secondary 30C15, 33C52.

Key words and phrases. Jacobi polynomials, generalized Laguerre polynomials, multiplier sequences, $n$-sequences, positive pairs.

(C)2010 American Mathematical Society Reverts to public domain 28 years from publication 
The Hermite-Biehler theorem ([13, [24, p. 197]) states that the even and odd parts of stable polynomials form positive pairs. This supplies us with a powerful tool to prove reality and negativity of the zeros of certain polynomials. In addition, we will also make use of the following characterization of positive pairs.

Lemma 3 (D. Wagner [26, Lemma 2.4]). Let $P(z)$ and $Q(z)$ be real standard polynomials (i.e., the leading coefficients of the real polynomials $P$ and $Q$ are positive) with only negative zeros. Then $P(z)$ interlaces (or alternates) $Q(z)$ (in the sense of Definition 1) if and only if for all $\mu>0$ both $Q(z)+\mu P(z)$ and $Q(z)+\mu z P(z)$ have only real negative zeros.

An outline of this paper is as follows. We prove that polynomials whose coefficients are successive derivatives of a class of generalized Laguerre polynomials (evaluated at $x=0$ ) are stable (Theorem 4). This result is sharp (cf. Remark 1; see also Conjecture 1 and Open Problem 1). More general combinations of these polynomials are also stable (Theorem 8 ). We also show that a related family of polynomials possess only real negative zeros (Theorem 5 and Theorem 9 ). With the aid of the aforementioned results, together with the Hermite-Biehler Theorem and Lemma 3, we show that some members of the above family (depending on a parameter) form positive pairs (Theorem 10). In Section 3, we consider a special class of stability-preserving operators, known as multiplier sequences (Theorem 11). With the aid of $n$-sequences (Definition 12) and a recent result about the zero distribution of hypergeometric polynomials (Theorem 14), we prove that a certain family of stable polynomials have only real negative zeros.

\section{NeW Classes of STABle POLYNOMials AND POSITIVE Pairs}

In this section, we present a new class of stable polynomials obtained with the aid of the generalized Laguerre polynomials, $L_{n}^{(\alpha)}(x)$, where $L_{n}^{(\alpha)}$ can be defined via the Rodrigues formula (cf. [23, pp. 203-204]),

$$
L_{n}^{(\alpha)}(x):=\frac{x^{-\alpha} e^{x}}{n !} \frac{d^{n}}{d x^{n}}\left(e^{-x} x^{n+\alpha}\right)=\sum_{j=0}^{n}(-1)^{j}\left(\begin{array}{c}
n+\alpha \\
n-j
\end{array}\right) \frac{x^{j}}{j !} .
$$

Since $\frac{d^{k}}{d x^{k}} L_{n}^{(\alpha)}(x)=(-1)^{k} L_{n-k}^{(\alpha+k)}(x)$ (see, for example, [23, p. 203]), it follows from

(1) that $\left(\frac{d^{k}}{d x^{k}} L_{n}^{(\alpha)}(x)\right)_{x=0}=(-1)^{k}\left(\begin{array}{c}n+\alpha \\ n-k\end{array}\right)$ and whence

$$
\sum_{k=0}^{n}(-1)^{k} z^{k} D^{k} L_{n}^{(\alpha)}(0)=\sum_{k=0}^{n}\left(\begin{array}{c}
n+\alpha \\
n-k
\end{array}\right) z^{k}, \quad z \in \mathbb{C}, \quad D:=\frac{d}{d x}
$$

a relation we will use in the following theorem.

Theorem 4. For each $n \geq 1$, the polynomial

$$
p_{n}^{(\alpha)}(z)=\sum_{k=0}^{n}(-1)^{k} z^{k} D^{k} L_{n}^{(\alpha)}(0)=\sum_{k=0}^{n}\left(\begin{array}{l}
n+\alpha \\
n-k
\end{array}\right) z^{k} \quad\left(D:=\frac{d}{d x}\right)
$$

is stable for $-1<\alpha \leq 1$, where $L_{n}^{(\alpha)}$ denotes the generalized Laguerre polynomial of degree $n$ (cf. (1)). 
Proof. Consider the polynomial

$$
f_{n}(x ; z)=\sum_{k=0}^{n} z^{k} D^{k} L_{n}^{(\alpha)}(x) \quad(z \in \mathbb{C}, x \in \mathbb{R}),
$$

and note that for $z \neq 0, f_{n}$ satisfies the differential equation

$$
\frac{1}{z}\left(f_{n}-L_{n}^{(\alpha)}\right)=\frac{d f_{n}}{d x}
$$

Multiply (5) by $\frac{1}{x} \overline{f_{n}}$, and then add to this its complex conjugate to obtain

$$
\left(\frac{1}{z}+\frac{1}{\bar{z}}\right)\left|f_{n}\right|^{2} \frac{1}{x}-\left(\frac{\overline{f_{n}}}{x z}+\frac{f_{n}}{x \bar{z}}\right) L_{n}^{(\alpha)}=\frac{d\left|f_{n}\right|^{2}}{d x} \frac{1}{x} .
$$

Next, multiply both sides of ([6) by the (generalized) Laguerre weight $w(x)=x^{\alpha} e^{-x}$, and for any $\varepsilon>0$, integrate over $(\varepsilon, \infty)$ :

$$
\begin{aligned}
\left(\frac{1}{z}+\frac{1}{\bar{z}}\right) \int_{\varepsilon}^{\infty}\left|f_{n}\right|^{2} x^{\alpha-1} e^{-x} d x-\int_{\varepsilon}^{\infty}\left(\frac{\overline{f_{n}}}{x z}+\frac{f_{n}}{x \bar{z}}\right) & L_{n}^{(\alpha)} x^{\alpha} e^{-x} d x \\
& =\int_{\varepsilon}^{\infty} \frac{d\left|f_{n}\right|^{2}}{d x} x^{\alpha-1} e^{-x} d x .
\end{aligned}
$$

Now, let $z_{j}$ be any zero of $f_{n}(0 ; z)$ and note that $z_{j} \neq 0$. Since $f_{n}\left(0 ; z_{j}\right)=0$, it follows that $\frac{f_{n}\left(x ; z_{j}\right)}{x}$ is a polynomial of degree $n-1$ in $x$. Hence, as $\varepsilon \rightarrow 0^{+}$, the orthogonality property of the Laguerre polynomials for $\alpha>-1$ (cf. [23, p. 206]) implies that the second term of (7) vanishes. Furthermore, an integration by parts yields

$$
\frac{2 \operatorname{Re}\left(z_{j}\right)}{\left|z_{j}\right|^{2}} \int_{0}^{\infty}\left|f_{n}\right|^{2} x^{\alpha-1} e^{-x} d x=-\int_{0}^{\infty}\left|f_{n}\right|^{2}(\alpha-1-x) x^{\alpha-2} e^{-x} d x,
$$

where the existence of the improper integrals can be justified as was done above. Since $-1<\alpha \leq 1, x \geq 0$ and $\alpha-1-x \leq 0$, it follows from (8) that $\operatorname{Re}\left(z_{j}\right)>0$. We have thus shown that the real part of an arbitrary zero of $f_{n}(0 ; z)$ is positive and consequently, the zeros of the polynomial

$$
p_{n}^{(\alpha)}(z)=f_{n}(0 ;-z)=\sum_{k=0}^{n}(-1)^{k} z^{k} D^{k} L_{n}^{(\alpha)}(0) \quad(-1<\alpha \leq 1)
$$

all have negative real parts; that is, $p_{n}^{(\alpha)}$ is a stable polynomial.

We note that the polynomials $p_{n}^{(\alpha)}$ are hypergeometric polynomials which can be expressed in terms of Jacobi polynomials, where one of the parameters depends on $n$ (see, for example, [25, p. 62, eq. 4.21.2]):

$$
p_{n}^{(\alpha)}(z)=\left(\begin{array}{c}
n+\alpha \\
n
\end{array}\right){ }_{2} F_{1}(-n, 1 ; \alpha+1 ;-z)=P_{n}^{(\alpha,-n-\alpha)}(1+2 z) .
$$

Before we proceed to establish some more general results and consequences of Theorem 4, we pause here for a moment (see Remarks 1-3) and examine the need for the assumptions on the parameter $\alpha$ in Theorem 4, provide some examples, formulate a conjecture, propose an open problem related to Theorem 4 , and call attention to the Laplace transform representation of the polynomials $p_{n}^{(\alpha)}$. 
Remark 1 . We remark that Theorem 4 is sharp in the sense that the restriction on the parameter $\alpha$ is necessary. Indeed, if $\alpha$ lies outside the interval $(-1,1]$, then, in general, the polynomial $p_{n}^{(\alpha)}$ need not be stable. For example, with the aid of, say, Mathematica or Maple, one can readily verify that when $n=5$ and $\alpha=20$, the polynomial $p_{5}^{(20)}$ is not stable. Note that if $\alpha=-1$, then the requisite integrability condition in the proof of Theorem 2 is not satisfied. However, when $\alpha=-1$, then $p_{n}^{(-1)}(z)=z(1+z)^{n-1}, n \geq 2$, and whence $p_{n}^{(-1)}$ is only quasi-stable; that is, the zeros of $p_{n}^{(-1)}$ lie in the closed half-plane, $\operatorname{Re} z \leq 0$. In addition, we formulate the following conjecture that is based on our experiments and numerical work. Our preliminary work further suggests that the closer $\alpha$ is to 1 , where $\alpha>1$, the larger $n$ has to be to produce an unstable polynomial $p_{n}^{(\alpha)}$.

Conjecture 1. For each $\alpha>1$, there is a positive integer $n$ such that the polynomial $p_{n}^{(\alpha)}$ (cf. (3) of Theorem 4) is unstable.

Open Problem 1. If $p$ is a polynomial of degree $n$ with zeros $\left\{z_{k}\right\}_{k=1}^{n}$, where Re $z_{k}<0, k=1,2, \ldots, n$, the abscissa of stability of $p$ is defined as $\sigma(p):=$ $\max _{1 \mid \leq k \leq n} \operatorname{Re} z_{k}$. Determine the abscissa of stability of $p_{n}^{(\alpha)}$ (cf. (3) of Theorem 4 ) for $-1<\alpha \leq 1$.

Remark 2. We also note that if the real polynomial $p$ is stable, where $\operatorname{deg} p=n \geq 2$ and $p(0) \neq 0$, then so is the reverse polynomial $p^{*}(z):=z^{n} p\left(\frac{1}{z}\right)$. Indeed, $z_{k}$, $\operatorname{Re} z_{k}<0$, is a zero of $p$ if and only if $\frac{1}{z_{k}}$ is a zero of $p^{*}$ and $\operatorname{Re} \frac{1}{z_{k}}=\frac{\operatorname{Re} z_{k}}{\left|z_{k}\right|^{2}}<0$. Consequently, it follows from Theorem 4 (cf. (3)) that the polynomial

$$
b_{n}^{(\alpha)}(z):=\left(p_{n}^{(\alpha)}(z)\right)^{*}=\sum_{k=0}^{n}\left(\begin{array}{c}
n+\alpha \\
k
\end{array}\right) z^{k}
$$

is stable whenever $-1<\alpha \leq 1$. Now observe that $b_{n}^{(\alpha)}$, the $n^{\text {th }}$ partial sum of the binomial series $(1+z)^{n+\alpha},|z|<1$, is a stable polynomial for $-1<\alpha \leq 1$. We hasten to note that if $m \in \mathbb{N}, m \neq n$, then, in general, the $m^{t h}$ partial sum of the binomial series $(1+z)^{n+\alpha}$ is not a stable polynomial. For example, one can show that the $7^{\text {th }}$ partial sum of $(1+z)^{12}$ is not a stable polynomial. In Section 3 , we will reexamine this curious phenomenon in conjunction with the theory of multiplier sequences and $n$-sequences.

Remark 3. We also note here that Theorem 4 can be reformulated using some simple linear operators acting on the vector space, $\mathbb{R}[x]$, of all real polynomials. Let $T:=\left\{\frac{1}{k !}\right\}_{k=0}^{\infty}$ and $\Lambda:=\{k !\}_{k=0}^{\infty}$. Define the action of $T$ (and similarly the action of $\Lambda$ ) on monomials by $T\left[x^{k}\right]:=\frac{1}{k !} x^{k}$ and then extend the definition to $\mathbb{R}[x]$ by linearity. We note that $T$ preserves stability (see Section 3 ) while, in general, $\Lambda$ does not. Indeed, by way of illustration, consider the stable polynomial $f(x)=(1+x)^{6}$. Now, with the aid of some numerical work (or otherwise) we can demonstrate that $\Lambda\left[(1+x)^{6}\right]=\sum_{k=0}^{6}\left(\begin{array}{l}6 \\ k\end{array}\right) k ! x^{k}$ is not a stable polynomial. In contrast, we know from Theorem 4 that $\Lambda\left[L_{n}^{(\alpha)}(-x)\right]$ is a stable polynomial whenever $-1<\alpha \leq 1$. Or equivalently, we observe that, for $k=0,1,2, \ldots$,

$$
k !=\Gamma(k+1)=\int_{0}^{\infty} e^{-t} t^{k} d t \quad \text { and } \quad L_{n}^{(\alpha)}(-z t)=\sum_{j=0}^{n}\left(\begin{array}{c}
n+\alpha \\
n-k
\end{array}\right) \frac{t^{k} z^{k}}{k !} \quad(t>0),
$$


and thus, by Theorem 4 , if $-1<\alpha \leq 1$, then

$$
p_{n}^{(\alpha)}(z)=\Lambda\left[L_{n}^{(\alpha)}(-z)\right]=\int_{0}^{\infty} e^{-t} L_{n}^{(\alpha)}(-z t) d t=\sum_{k=0}^{n}\left(\begin{array}{c}
n+\alpha \\
n-k
\end{array}\right) z^{k}
$$

is a stable polynomial. Can one deduce directly from this elegant formula that $p_{n}^{(\alpha)}$ is a stable polynomial?

With the aid of the Hermite-Biehler Theorem and Theorem 4, we next obtain a family of polynomials all of whose zeros are real and negative.

Theorem 5. Let $L_{n}^{(\alpha)}$ denote the generalized Laguerre polynomial of degree $n$, $n \geq 2$. If $-1<\alpha \leq 2$, then the zeros of the polynomial

$$
q_{n}^{(\alpha)}(z)=\sum_{k=0}^{\left[\frac{n}{2}\right]} z^{k} D^{2 k} L_{n}^{(\alpha)}(0)=\sum_{k=0}^{\left[\frac{n}{2}\right]}\left(\begin{array}{c}
n+\alpha \\
n-2 k
\end{array}\right) z^{k}
$$

are all real and negative. Moreover, if $-1<\alpha \leq 1$, then the polynomials $q_{n}^{(\alpha)}$ and $q_{n-1}^{(\alpha+1)}$ form a positive pair.

Proof. Applying the Hermite-Biehler Theorem (cf. Theorem 2) to the stable polynomial $p_{n}^{(\alpha)},-1<\alpha \leq 1$ (cf. (3) of Theorem 4), we find that the zeros of the polynomials

$$
\Omega_{1, n}^{(\alpha)}(z):=\sum_{k=0}^{\left[\frac{n}{2}\right]} z^{k} D^{2 k} L_{n}^{(\alpha)}(0) \quad \text { and } \quad \Omega_{2, n}^{(\alpha)}(z):=\sum_{k=0}^{\left[\frac{n}{2}\right]} z^{k} D^{2 k+1} L_{n}^{(\alpha)}(0)
$$

are all real and negative. Since $\Omega_{1, n}^{(\alpha)}=q_{n}^{(\alpha)}$, the theorem is verified for $-1<\alpha \leq 1$. Next, using the relation $D L_{n}^{(\alpha)}(0)=-L_{n-1}^{(\alpha+1)}(0)(D=d / d x)$, we obtain that

$$
\Omega_{2, n}^{(\alpha)}(z)=\sum_{k=0}^{\left[\frac{n}{2}\right]} z^{k} D^{2 k+1} L_{n}^{(\alpha)}(0)=-\sum_{k=0}^{\left[\frac{n}{2}\right]} z^{k} D^{2 k} L_{n-1}^{(\alpha+1)}(0)=-q_{n-1}^{(\alpha+1)}(z)
$$

has only real negative zeros for $-1<\alpha \leq 1$. Therefore, the change of indices, $n \rightarrow n+1$ and $\alpha \rightarrow \alpha-1$, shows that all the zeros of the polynomial $q_{n}^{(\alpha)}$ are real and negative for $0<\alpha \leq 2$. Since $\Omega_{1, n}^{(\alpha)}=q_{n}^{(\alpha)}$ and $\Omega_{2, n}^{(\alpha)}=-q_{n-1}^{(\alpha+1)}$ (cf. (12)), the last assertion of the theorem is an immediate consequence of Theorem 2. This completes the proof.

It is well-known (12, [13]) that polynomial approximations to solutions of differential equations are the basic ingredient of many numerical methods. In 4, M. Charalambides and F. Waleffe point out the advantages of approximations based on orthogonal polynomials. In particular, these authors show that the characteristic polynomials for the Gegenbauer Tau method obey a three-term recurrence relation plus a constant term which vanishes for Legendre Tau and Galerkin cases. Inspired by the importance of the aforementioned results, we now proceed to show that the polynomials defined in Theorem 5 (cf. (10)) also obey a three-term recurrence relation plus a constant term. 
Theorem 6. The polynomials $q_{n}^{(\alpha)},-1<\alpha \leq 2$, defined by (10) of Theorem 5 ,

$$
q_{n}^{(\alpha)}(z)=\sum_{k=0}^{\left[\frac{n}{2}\right]} z^{k} D^{2 k} L_{n}^{(\alpha)}(0)=\sum_{k=0}^{\left[\frac{n}{2}\right]}\left(\begin{array}{c}
n+\alpha \\
n-2 k
\end{array}\right) z^{k},
$$

obey the following recurrence relation:

$$
z q_{n}^{(\alpha)}(z)=q_{n+2}^{(\alpha)}(z)-2 q_{n+1}^{(\alpha)}(z)+q_{n}^{(\alpha)}(z)-C_{n}^{(\alpha)},
$$

where

$$
C_{n}^{(\alpha)}=\left(\begin{array}{c}
n+\alpha \\
n
\end{array}\right) \frac{\alpha(\alpha-1)}{(n+1)(n+2)} .
$$

Proof. The generalized Laguerre polynomials satisfy the relation ([23, p. 202]), $L_{n}^{(\alpha)}(x)=-\frac{d}{d x} L_{n+1}^{(\alpha)}(x)+\frac{d}{d x} L_{n}^{(\alpha)}(x)$ and hence, in terms of the second derivatives, we have

$$
L_{n}^{(\alpha)}(x)=\frac{d^{2}}{d x^{2}} L_{n+2}^{(\alpha)}(x)-2 \frac{d^{2}}{d x^{2}} L_{n+1}^{(\alpha)}(x)+\frac{d^{2}}{d x^{2}} L_{n}^{(\alpha)}(x) .
$$

Substituting (16) into (13) and setting $q_{0}^{(\alpha)}(z)=1$, we obtain

$$
z q_{n}^{(\alpha)}(z)=q_{n+2}^{(\alpha)}(z)-2 q_{n+1}^{(\alpha)}(z)+q_{n}^{(\alpha)}(z)-C_{n}^{(\alpha)}, \quad \text { where }
$$

$C_{0}^{(\alpha)}=L_{2}^{(\alpha)}(0)=\left(\begin{array}{c}2+\alpha \\ 2\end{array}\right)$,

$C_{1}^{(\alpha)}=L_{3}^{(\alpha)}(0)-2 L_{2}^{(\alpha)}(0)=\left(\begin{array}{c}3+\alpha \\ 3\end{array}\right)-2\left(\begin{array}{c}2+\alpha \\ 2\end{array}\right)=\frac{(\alpha-3)(\alpha+1)(\alpha+2)}{6}, \quad$ and by induction

$C_{n}^{(\alpha)}=L_{n+2}^{(\alpha)}(0)-2 L_{n+1}^{(\alpha)}(0)+L_{n}^{(\alpha)}(0)=\cdots=\left(\begin{array}{c}n+\alpha \\ n\end{array}\right) \frac{\alpha(\alpha-1)}{(n+1)(n+2)}$.

Since $C_{n}^{(\alpha)}$ is zero if $\alpha=0$ or $\alpha=1$, we see that the polynomials $q_{n}^{(0)}$ and $q_{n}^{(1)}$ satisfy a three-term recurrence relation.

Remark 4. We note here, parenthetically, that an elementary induction argument shows that for $n=1,2,3, \ldots$,

$$
\begin{gathered}
q_{n}^{(0)}(z)=\sum_{k=0}^{\left[\frac{n}{2}\right]}\left(\begin{array}{c}
n \\
n-2 k
\end{array}\right) z^{k}=\frac{(1+\sqrt{z})^{n}+(1-\sqrt{z})^{n}}{2}, \\
q_{n}^{(1)}(z)=\sum_{k=0}^{\left[\frac{n}{2}\right]}\left(\begin{array}{c}
n+1 \\
n-2 k
\end{array}\right) z^{k}=\frac{(1+\sqrt{z})^{n+1}-(1-\sqrt{z})^{n+1}}{2 \sqrt{z}},
\end{gathered}
$$

with due regard for the removable singularity at $z=0$. Now, if $c_{n}:=a^{n}+b^{n}$, $a, b \in \mathbb{C}, n \in \mathbb{N}$, then $c_{n+2}-2 c_{n+1}+c_{n}=(-1+a)^{2} a^{n}+(-1+b)^{2} b^{n}$. Substituting $a \rightarrow$ $(1+\sqrt{z})$ and $b \rightarrow(1-\sqrt{z})$ in this expression, a calculation shows that $q_{n}^{(0)}$ satisfies a three-term recurrence relation. An analogous argument demonstrates, without appealing to the properties of the Laguerre polynomials, that the polynomials $q_{n}^{(1)}$ also satisfy a three-term recurrence relation.

As we shall see below, the family of polynomials described in Theorem 5 enjoys an additional significant property; polynomials of successive degrees form positive pairs. In order to prove this, we need to establish a number of results and we begin here with the following useful preparatory lemma. 
Lemma 7. Let $\lambda, \mu \geq 0$. For fixed $\alpha,-1<\alpha \leq 1$, set

$$
f_{n}(x ; z):=\sum_{k=0}^{n} z^{k} D^{k} L_{n}^{(\alpha)}(x)+\left(\lambda+\mu z^{2}\right) \sum_{k=0}^{n-1} z^{k} D^{k} L_{n-1}^{(\alpha)}(x) \quad(x \in \mathbb{R}, z \in \mathbb{C}),
$$

where $L_{n}^{(\alpha)}$ denotes the generalized Laguerre polynomial of degree $n \geq 2$ (cf. (11)). If $z_{j}$ is a zero of the polynomial $f_{n}(0 ; z)$, then

$$
I_{1}:=\int_{0}^{\infty}\left(\frac{\overline{f_{n}}}{x z_{j}}+\frac{f_{n}}{x \overline{z_{j}}}\right) L_{n-1}^{(\alpha)} x^{\alpha} e^{-x} d x=-\frac{1}{n}\left(\frac{1}{z_{j}}+\frac{1}{\overline{z_{j}}}\right) \frac{\Gamma(n+\alpha)}{(n-1) !}
$$

and

$$
I_{2}:=\int_{0}^{\infty}\left(\frac{z_{j} \overline{f_{n}}}{x}+\frac{\overline{z_{j}} f_{n}}{x}\right) L_{n-1}^{(\alpha)} x^{\alpha} e^{-x} d x=-\frac{1}{n}\left(z_{j}+\overline{z_{j}}\right) \frac{\Gamma(n+\alpha)}{(n-1) !},
$$

where $\Gamma$ denotes the gamma function and $f_{n}=f_{n}\left(x ; z_{j}\right)$.

Proof. From (20) it follows that

$$
f_{n}\left(x ; z_{j}\right)=L_{n}^{(\alpha)}(x)+h_{n-1}(x), \quad\left(h_{n-1}(x) \in \mathbb{C}[x], \operatorname{deg}\left(h_{n-1}\right) \leq n-1\right),
$$

and since $f_{n}\left(0 ; z_{j}\right)=0$, the polynomial $f_{n}\left(x ; z_{j}\right)$ can also be expressed as

$$
f_{n}\left(x ; z_{j}\right)=x \sum_{k=0}^{n-1} c_{k} L_{k}^{(\alpha)}(x)=x c_{n-1} L_{n-1}^{(\alpha)}(x)+\tilde{h}_{n-1}(x),
$$

where $\tilde{h}_{n-1}(x) \in \mathbb{C}[x], \operatorname{deg}\left(\tilde{h}_{n-1}\right) \leq n-1$. Next, we use the recurrence relation satisfied by the Laguerre polynomials (cf. [23, p. 202]),

$$
n L_{n}^{(\alpha)}(x)=(2 n-1+\alpha-x) L_{n-1}^{(\alpha)}(x)-(n-1+\alpha) L_{n-2}^{(\alpha)}(x),
$$

in conjunction with (24) and obtain that

(25) $f_{n}\left(x ; z_{j}\right)=-n c_{n-1} L_{n}^{(\alpha)}(x)+\hat{h}_{n-1}(x), \quad\left(\hat{h}_{n-1}(x) \in \mathbb{C}[x], \operatorname{deg}\left(\hat{h}_{n-1}\right) \leq n-1\right)$.

A comparison of (23) and (25) shows that $c_{n-1}=-\frac{1}{n}$. From (24) and the fact that $f_{n}\left(0 ; z_{j}\right)=0$, we deduce that, for $x \neq 0$,

(26) $\frac{f_{n}\left(x ; z_{j}\right)}{x}=-\frac{1}{n} L_{n-1}^{(\alpha)}(x)+r_{n-2}(x), \quad\left(r_{n-2}(x) \in \mathbb{C}[x], \operatorname{deg}\left(r_{n-2}\right) \leq n-2\right)$.

Thus, $\operatorname{deg}\left(\frac{\overline{f_{n}}\left(x ; z_{j}\right)}{x z_{j}}\right)=\operatorname{deg}\left(\frac{f_{n}\left(x ; z_{j}\right)}{x \bar{z}_{j}}\right)=n-1$. Now, using the orthogonality properties of the generalized Laguerre polynomials $L_{n}^{(\alpha)}$ with respect to the weight function $w(x)=x^{\alpha} e^{-x}$ (see, for example, [23, p. 206, formulae (7) and (8)]), we obtain

$$
\int_{0}^{\infty} r_{n-2}(x) L_{n-1}^{(\alpha)} x^{\alpha} e^{-x} d x=0
$$

and

$$
I_{1}=\int_{0}^{\infty}\left(\frac{\overline{f_{n}}}{x z_{j}}+\frac{f_{n}}{x \overline{z_{j}}}\right) L_{n-1}^{(\alpha)} x^{\alpha} e^{-x} d x=-\frac{1}{n}\left(\frac{1}{z_{j}}+\frac{1}{\overline{z_{j}}}\right) \frac{\Gamma(n+\alpha)}{(n-1) !} .
$$

Finally, the foregoing argument also shows that

$$
\int_{0}^{\infty}\left(\frac{\overline{f_{n}}}{x \bar{z}_{j}}+\frac{f_{n}}{x z_{j}}\right) L_{n-1}^{(\alpha)} x^{\alpha} e^{-x} d x=-\frac{1}{n}\left(\frac{1}{z_{j}}+\frac{1}{\overline{z_{j}}}\right) \frac{\Gamma(n+\alpha)}{(n-1) !} .
$$


Hence, multiplying both sides of (27) by $\left|z_{j}\right|^{2}$ yields the desired result:

$$
I_{2}=\int_{0}^{\infty}\left(\frac{z_{j} \overline{f_{n}}}{x}+\frac{\overline{z_{j}} f_{n}}{x}\right) L_{n-1}^{(\alpha)} x^{\alpha} e^{-x} d x=-\frac{1}{n}\left(z_{j}+\overline{z_{j}}\right) \frac{\Gamma(n+\alpha)}{(n-1) !} .
$$

Theorem 8. Let $p_{n}^{(\alpha)}$ denote the polynomial defined by (3) (see Theorem 4). If $\lambda, \mu \geq 0$, then the polynomial

$$
P_{n}(z ; \alpha, \lambda, \mu):=p_{n}^{(\alpha)}(z)+\left(\lambda+\mu z^{2}\right) p_{n-1}^{(\alpha)}(z)
$$

is stable for $-1<\alpha \leq 1$.

Proof. For fixed $\lambda, \mu \geq 0$ and $\alpha,-1<\alpha \leq 1$, consider

$$
f_{n}(x ; z):=\sum_{k=0}^{n} z^{k} D^{k} L_{n}^{(\alpha)}(x)+\left(\lambda+\mu z^{2}\right) \sum_{k=0}^{n-1} z^{k} D^{k} L_{n-1}^{(\alpha)}(x) \quad(x \in \mathbb{R}, z \in \mathbb{C}),
$$

where $L_{n}^{(\alpha)}$ denotes the generalized Laguerre polynomial of degree $n \geq 2$ (cf. (11)). Note that for $z \neq 0, f_{n}$ satisfies the differential equation

$$
\frac{1}{z}\left(f_{n}-L_{n}^{(\alpha)}-\left(\lambda+\mu z^{2}\right) L_{n-1}^{(\alpha)}\right)=\frac{d f_{n}}{d x} .
$$

Now, let $z_{j}$ be any zero of $f_{n}(0 ; z)$ and note that $z_{j} \neq 0$. Next, substitute $z_{j}$ for $z$ in (30), multiply both sides of (30) by $\frac{\overline{f_{n}\left(x ; z_{j}\right)}}{x} x^{\alpha} e^{-x}$, add the complex conjugate of the resulting expression and then integrate:

$$
\begin{aligned}
\left(\frac{1}{z_{j}}+\frac{1}{\overline{z_{j}}}\right) & \int_{0}^{\infty}\left|f_{n}\right|^{2} x^{\alpha-1} e^{-x} d x-\int_{0}^{\infty}\left(\frac{\overline{f_{n}}}{\overline{z_{j} x}}+\frac{f_{n}}{\overline{z_{j}} x}\right)\left(L_{n}^{(\alpha)}+\lambda L_{n-1}^{(\alpha)}\right) x^{\alpha} e^{-x} d x \\
& -\mu \int_{0}^{\infty}\left(\frac{z_{j} \overline{f_{n}}}{x}+\frac{\overline{z_{j}} f_{n}}{x}\right) L_{n-1}^{(\alpha)} x^{\alpha} e^{-x} d x=\int_{0}^{\infty} \frac{d\left|f_{n}\right|^{2}}{d x} x^{\alpha-1} e^{-x} d x
\end{aligned}
$$

where $f_{n}=f_{n}\left(x ; z_{j}\right)$. Since $f_{n}\left(0 ; z_{j}\right)=0, \frac{f_{n}\left(x ; z_{j}\right)}{x}$ and its complex conjugate are polynomials of degree $n-1$ in $x$. Thus, by orthogonality of $L_{n}^{(\alpha)}$ with respect to the weight function $w(x)=x^{\alpha} e^{-x}$,

$$
I_{0}=\int_{0}^{\infty}\left(\frac{\overline{f_{n}}}{x z_{j}}+\frac{f_{n}}{x \overline{z_{j}}}\right) L_{n}^{(\alpha)} x^{\alpha} e^{-x} d x=0 .
$$

Therefore, with the aid of (21) and (22) in Lemma 2 (cf. $I_{1}$ and $I_{2}$ ), we can express (31) in the form

$$
\left(\frac{1}{z_{j}}+\frac{1}{\overline{z_{j}}}\right) \int_{0}^{\infty}\left|f_{n}\right|^{2} x^{\alpha-1} e^{-x} d x-\lambda I_{1}-\mu I_{2}=\int_{0}^{\infty} \frac{d\left|f_{n}\right|^{2}}{d x} x^{\alpha-1} e^{-x} d x
$$

or, using the explicit expressions for $I_{1}$ and $I_{2}$ and simplifying, we have

$$
\begin{aligned}
\left(\frac{1}{z_{j}}+\frac{1}{\overline{z_{j}}}\right)\left[\int_{0}^{\infty}\left|f_{n}\right|^{2} x^{\alpha-1} e^{-x} d x+\frac{1}{n} \frac{\Gamma(n+\alpha)}{(n-1) !}\left(\lambda+\mu\left|z_{j}\right|^{2}\right)\right] \\
=\int_{0}^{\infty} \frac{d\left|f_{n}\right|^{2}}{d x} x^{\alpha-1} e^{-x} d x .
\end{aligned}
$$


Further simplification, together with an integration by parts, yields

$$
\begin{array}{r}
\frac{2 \operatorname{Re}\left(z_{j}\right)}{\left|z_{j}\right|^{2}}\left[\int_{0}^{\infty}\left|f_{n}\right|^{2} x^{\alpha-1} e^{-x} d x+\frac{\Gamma(n+\alpha)}{n !}\left(\lambda+\mu\left|z_{j}\right|^{2}\right)\right] \\
=-\int_{0}^{\infty}\left|f_{n}\right|^{2}(\alpha-1-x) x^{\alpha-2} e^{-x} d x .
\end{array}
$$

If $-1<\alpha \leq 1$ and $x \geq 0$, then $\alpha-1-x \leq 0$ and whence $\operatorname{Re}\left(z_{j}\right)>0$, by (32). Therefore, if $\lambda, \mu \geq 0$, then the polynomial

$$
\begin{aligned}
f_{n}(0 ;-z) & =\sum_{k=0}^{n}(-1)^{k} z^{k} D^{k} L_{n}^{(\alpha)}(0)+\left(\lambda+\mu z^{2}\right) \sum_{k=0}^{n-1}(-1)^{k} z^{k} D^{k} L_{n-1}^{(\alpha)}(0) \\
& =\sum_{k=0}^{n}\left(\begin{array}{c}
n+\alpha \\
n-k
\end{array}\right) z^{k}+\left(\lambda+\mu z^{2}\right) \sum_{k=0}^{n-1}\left(\begin{array}{c}
n-1+\alpha \\
n-1-k
\end{array}\right) z^{k} \\
& =p_{n}^{(\alpha)}(z)+\left(\lambda+\mu z^{2}\right) p_{n-1}^{(\alpha)}(z) \\
& =P_{n}(z ; \alpha, \lambda, \mu)
\end{aligned}
$$

is stable for $-1<\alpha \leq 1$.

From the above theorem we deduce the following result.

Theorem 9. Let

$$
q_{n}^{(\alpha)}(z)=\sum_{k=0}^{\left[\frac{n}{2}\right]} z^{k} D^{2 k} L_{n}^{(\alpha)}(0)=\sum_{k=0}^{\left[\frac{n}{2}\right]}\left(\begin{array}{c}
n+\alpha \\
n-2 k
\end{array}\right) z^{k} .
$$

If $\lambda, \mu \geq 0$, then the zeros of the polynomial

$$
Q_{n}(z ; \alpha, \lambda, \mu):=q_{n}^{(\alpha)}(z)+(\lambda+\mu z) q_{n-1}^{(\alpha)}(z) \quad(n \geq 2)
$$

are all real and negative for $-1<\alpha \leq 2$.

Proof. Fix $\lambda, \mu \geq 0$. By Theorem 8 , the polynomial

$$
P_{n}(z ; \alpha, \lambda, \mu):=p_{n}^{(\alpha)}(z)+\left(\lambda+\mu z^{2}\right) p_{n-1}^{(\alpha)}(z)
$$

is stable for $-1<\alpha \leq 1$. Now a calculation shows that $P_{n}(z ; \alpha, \lambda, \mu)$ can be expressed as

$$
P_{n}(z ; \alpha, \lambda, \mu)=\Omega_{1, n}^{(\alpha)}\left(z^{2}\right)+z \Omega_{2, n-1}^{(\alpha+1)}\left(z^{2}\right)
$$

where

$$
\begin{gathered}
\Omega_{1, n}^{(\alpha)}(z):=q_{n}^{(\alpha)}(z)+(\lambda+\mu z) q_{n-1}^{(\alpha)}(z) \quad \text { and } \\
\Omega_{2, n-1}^{(\alpha+1)}(z):=q_{n-1}^{(\alpha+1)}(z)+(\lambda+\mu z) q_{n-2}^{(\alpha+1)}(z) .
\end{gathered}
$$

Hence, by the Hermite-Biehler Theorem, the zeros of $\Omega_{1, n}^{(\alpha)}(z)=Q_{n}(z ; \alpha, \lambda, \mu)$ are all real and negative for $-1<\alpha \leq 1$. Since the zeros of $\Omega_{2, n-1}^{(\alpha+1)}$ are also all real and negative for $-1<\alpha \leq 1$, the change of indices, $n \rightarrow n+1$ and $\alpha \rightarrow \alpha-1$, shows that the zeros of $Q_{n}(z ; \alpha, \lambda, \mu)$ are all real and negative for $0<\alpha \leq 2$.

As an immediate consequence of Theorem 9 and Lemma 3, we obtain the following principal result of this section. 
Theorem 10. If $-1<\alpha \leq 2$, then the polynomials $q_{n}^{(\alpha)}$ and $q_{n-1}^{(\alpha)}$ (defined in (34) of Theorem 9) form a positive pair.

Remark 5. Asymptotic results for Jacobi polynomials with negative parameters have been obtained recently. From these results one may derive conclusions about the distribution of zeros when $n \rightarrow \infty$ (see, for example, A. B. J. Kuijlaars and A. Martinez-Finkelshtein [16], A. B J. Kuijlaars, A. Martinez-Finkelshtein and R. Orive [17, A. Martinez-Finkelshtein and R. Orive [20]).

\section{Scholia. multiplier Sequences, $n$-Sequences And Stability}

Linear operators that preserve stability play an important role in stability analysis. Recently, in a series of papers ([1], [2], 3]), J. Borcea and P. Brändén obtained a complete characterization of all linear operators that preserve stability of univariate as well as multivariate polynomials. Here, our focus will be on some special classes of stability-preserving linear operators as they pertain to the results obtained in Section 2 (cf. Remark 3). By Theorem 4 and Remark 2, the polynomial $b_{n}^{(\alpha)}(z)=\sum_{k=0}^{n}\left(\begin{array}{c}n+\alpha \\ k\end{array}\right) z^{k}$ is stable for $-1<\alpha \leq 1$. Since the Hadamard product of stable polynomials is stable (J. Garloff and D.G. Wagner [14]), it follows that the polynomial

$$
B_{n}^{(\alpha)}(z):=b_{n}^{(\alpha)}(z) *(1+z)^{n}:=\sum_{k=0}^{n}\left(\begin{array}{l}
n \\
k
\end{array}\right)\left(\begin{array}{c}
n+\alpha \\
k
\end{array}\right) z^{k}
$$

is stable for $-1<\alpha \leq 1$. But more is true, for we will show that if $-1<\alpha$, then the polynomials $B_{n}^{(\alpha)}, n=1,2,3, \ldots$, have only real and negative zeros.

Remark 6. If $\alpha=m$ is a non-negative integer, then it is known, and in any case it is easy to prove, that $B_{n}^{(m)}, \operatorname{deg} B_{n}^{(m)}(z)=\min (m, n)$, has only real negative zeros. Rather than elaborate here on an elementary proof, we justify the aforementioned claim by noting that it is an immediate consequence of the Schur-Szegö-Malo Composition Theorem [21, Satz 7.5, p. 29]. We remark that the polynomials $B_{n}^{(\alpha)}$ are hypergeometric polynomials which can be expressed in terms of Jacobi polynomials, where one of the parameters depends on $n$ (see, for example, [25, p. 62, eq. 4.21.2]):

$$
B_{n}^{(\alpha)}(z)={ }_{2} F_{1}(-n,-n-\alpha ; 1 ; z)=P_{n}^{(0,-2 n-\alpha-1)}(1-2 z) .
$$

In order to motivate our proof, we will digress here for a moment and succinctly summarize some facts from the theory of multiplier sequences. To begin with, we recall the following terminology. A sequence $T=\left\{\gamma_{k}\right\}_{k=0}^{\infty}$ of non-negative real numbers is called a multiplier sequence if, whenever the real polynomial $p(z)=$ $\sum_{k=0}^{n} a_{k} z^{k}$ has only real zeros, the polynomial $T[p(z)]:=\sum_{k=0}^{n} \gamma_{k} a_{k} z^{k}$ also has only real zeros. (For a survey of results pertaining to multiplier sequences, see 8 and the references contained therein.) The algebraic characterization of multiplier sequences [22, p. 111] asserts that $T=\left\{\gamma_{k}\right\}_{k=0}^{\infty}, \gamma_{k} \geq 0$, is a multiplier sequence if and only if the polynomial

$g_{m}(z):=T\left[(1+z)^{m}\right]=\sum_{j=0}^{m}\left(\begin{array}{c}m \\ j\end{array}\right) \gamma_{j} z^{j} \quad$ has only real zeros for all $m=1,2,3, \ldots$ 
The polynomials $g_{m}(z)$ are called the Jensen polynomials associated with $T$. The transcendental characterization of multiplier sequences may be expressed as follows. Let $\varphi(z):=\sum_{k=0}^{\infty} \gamma_{k} \frac{z^{k}}{k !}, \gamma_{k} \geq 0$, be a real entire function of order at most one. Then the Taylor coefficients $\left\{\gamma_{k}\right\}_{k=0}^{\infty}$ form a multiplier sequence if and only if $\varphi(z)$ has only real negative zeros ([18, Ch. VIII], [22, p. 115], [9] or [7]). Another fundamental result states that if $\varphi$ is an entire function of order at most one having only real negative zeros, then the sequence $T=\{\varphi(k)\}_{k=0}^{\infty}$ is a multiplier sequence by a theorem of Laguerre (cf. [21, Satz 3.2] or [7, Theorem 1.4]). One of the simplest stability-preserving operators is the differentiation operator, $D:=d / d z$, by virtue of the Gauss-Lucas Theorem ([19, p. 12], [9]). The next theorem says that multiplier sequences are also stability-preserving operators.

Theorem $11([9])$. Let $T=\left\{\gamma_{k}\right\}_{k=0}^{\infty}$ be a non-negative multiplier sequence. Then $T[p(z)]:=\sum_{k=0}^{n} a_{k} \gamma_{k} z^{k}$ is a stable polynomial whenever $p(z)=\sum_{k=0}^{n} a_{k} z^{k}$ is a stable polynomial.

The paradigm example of a non-trivial multiplier sequence is the sequence $T=$ $\left\{\frac{1}{k !}\right\}_{k=0}^{\infty}=\left\{\frac{1}{\Gamma(k+1)}\right\}_{k=0}^{\infty}$. Since $p_{n}^{(\alpha)}(z)=\sum_{k=0}^{n}\left(\begin{array}{c}n+\alpha \\ k\end{array}\right) z^{k},-1<\alpha \leq 1$, is stable, it follows from Theorem 11 that the polynomial $T\left[p_{n}^{(\alpha)}(z)\right]=\sum_{k=0}^{n}\left(\begin{array}{c}n+\alpha \\ k\end{array}\right) \frac{z^{k}}{k !}$, $-1<\alpha \leq 1$, is also a stable polynomial. However, simple examples show that the polynomial $T\left[p_{n}^{(\alpha)}(z)\right]$, where $T=\left\{\frac{1}{k !}\right\}_{k=0}^{\infty}$ need not have only real zeros. (For example, set $n=7$ and $\alpha=-1 / 2$.) These considerations and examples suggest that we generalize the notion of a multiplier sequence as follows.

Definition 12. For a fixed positive integer $n$, let $T=\left\{\gamma_{0}, \gamma_{1}, \ldots, \gamma_{n}\right\}, \gamma_{k} \geq 0$, $k=0,1, \ldots, n$. Then $T$ is called an $n$-sequence if for every real polynomial $p(z)$, $\operatorname{deg} p(z) \leq n$, with only real zeros, the polynomial $T[p(z)]$ also has only real zeros.

Thus, it is clear from the definition that every multiplier sequence is an $n$ sequence (for all $n \in \mathbb{N}$ ), but that, in general, an $n$-sequence need not be a multiplier sequence. Today, there is no known transcendental characterization of $n$-sequences, but there is a useful algebraic characterization that is similar to the one cited above for multiplier sequences.

Proposition $13([6])$. Let $T=\left\{\gamma_{0}, \gamma_{1}, \ldots, \gamma_{n}\right\}, \gamma_{k} \geq 0$. Then $T$ is an n-sequence if and only if the polynomial $T\left[(1+z)^{n}\right]$ has only real zeros.

With the terminology adopted here, our goal is to prove that, for $-1<\alpha$, the sequence $T=\left\{\left(\begin{array}{c}n+\alpha \\ k\end{array}\right)\right\}_{k=0}^{n}$ is an $n$-sequence. Thus, in light of the above proposition, we need to show that the polynomial $B_{n}^{(\alpha)}$ (cf. (40)) has only real negative zeros. For the reader's convenience we also recall here a few pertinent facts about the Gauss hypergeometric function, ${ }_{2} F_{1}$, defined by

$$
{ }_{2} F_{1}(a, b ; c ; z)=1+\sum_{k=1}^{\infty} \frac{(a)_{k}(b)_{k}}{(c)_{k}} \frac{z^{k}}{k !}, \quad|z|<1,
$$

where $a, b$ and $c$ are complex parameters and $(\alpha)_{k}=\alpha(\alpha+1) \cdots(\alpha+k-1)=$ $\Gamma(\alpha+k) / \Gamma(\alpha)$ is the Pochhammer symbol. If $\alpha=-n$ is a negative integer, the series terminates and reduces to a polynomial of degree $n$, called a hypergeometric polynomial. Since $(-n)_{k}=(-1)^{k} n ! /(n-k)$ ! if $0 \leq k \leq n,(-n)_{k}=0$ if $k \geq n+1$, 
we find that

(43) ${ }_{2} F_{1}(-n,-n-\alpha ; 1 ; z)=1+\sum_{k=1}^{n} \frac{(-n)_{k}(-n-\alpha)_{k}}{k !} \frac{z^{k}}{k !}=\sum_{k=0}^{n}\left(\begin{array}{l}n \\ k\end{array}\right)\left(\begin{array}{c}n+\alpha \\ k\end{array}\right) z^{k}$.

Using the Hilbert-Klein formulae [25, Theorem 7.2, p. 145] and the connection between hypergeometric polynomials, ${ }_{2} F_{1}$, and the Jacobi polynomials, K. Driver and K. Jordaan [11] recently obtained the following result concerning the zero distribution of hypergeometric polynomials.

Theorem 14 ([11, Theorem 3.2 (iv), (v)]). Fix $b, c \in \mathbb{R}$, where $c>0$, and set $F(z)={ }_{2} F_{1}(-n, b ; c ; z)$.

(i) If $b<-n$, then all the zeros of $F(z)$ are real and negative.

(ii) If $-n<b<0,-j<b<-j+1, j=1,2, \ldots, n$, then $F(z)$ has $j$ real negative zeros. The remaining $(n-j)$ zeros of $F$ are all non-real if $(n-j)$ is even, while if $(n-j)$ is odd, $F$ has $(n-1-j)$ non-real zeros and one additional zero in $(1, \infty)$.

Preliminaries aside, we are now in a position to show that the polynomial $B_{n}^{(\alpha)}$ has only real negative zeros. If $\alpha$ is not an integer, this establishes the existence of (what appears to be) a new family of $n$-sequences.

Theorem 15. Let $n \geq 2$. If $-1<\alpha$, then the polynomial $B_{n}^{(\alpha)}$ has only real negative zeros and whence the sequence $T=\left\{\left(\begin{array}{c}n+\alpha \\ k\end{array}\right)\right\}_{k=0}^{n}$ is an n-sequence.

Proof. First we note that for $\alpha>-1$ and $0 \leq k \leq n,\left(\begin{array}{c}n+\alpha \\ k\end{array}\right) \geq 0$. Thus, by Proposition 13, it suffices to show that the polynomial

$$
B_{n}^{(\alpha)}(z):=\sum_{k=0}^{n}\left(\begin{array}{l}
n \\
k
\end{array}\right)\left(\begin{array}{c}
n+\alpha \\
k
\end{array}\right) z^{k}
$$

has only real zeros. Set $c=1$ and $b=-n-\alpha$. Now $B_{n}^{(\alpha)}(z)={ }_{2} F_{1}(-n,-n-\alpha ; 1 ; z)$ (cf. (43)). If $\alpha>0$, then $b<-n$, and hence by Theorem 14 (i), $B_{n}^{(\alpha)}$ has only real negative zeros. If $-1<\alpha<0$, then $b=-n-\alpha<-n+1$, and whence by part (ii)

of Theorem 14 (with $j=n$ ), $B_{n}^{(\alpha)}$ has only real negative zeros. Finally, $B_{n}^{(0)}$ has only real negative zeros, since the sequence $\left\{\left(\begin{array}{l}n \\ k\end{array}\right)\right\}_{k=0}^{n}$ is a multiplier sequence (cf. Remark 5).

\section{ACKNOWLEDGMEnT}

The authors thank the referee for a careful reading of the manuscript.

\section{REFERENCES}

[1] J. Borcea and P. Brändén, Pólya-Schur master theorems for circular domains and their boundaries, Ann. of Math. (2) 170 (2009), 465-492. arXiv: math.CV/0607416. MR2521123

[2] J. Borcea and P. Brändén, The Lee-Yang and Pólya-Schur programs. I. Linear operators preserving stability, Invent. Math. 177 (2009), 541-569. arXiv: 0809.0401. MR2534100

[3] J. Borcea and P. Brändén, The Lee-Yang and Pólya-Schur programs. II. Theory of stable polynomials and applications, Comm. Pure Appl. Math. 62 (2009), 1595-1631. arXiv: 0809.3087. MR2569072

[4] M. Charalambides and F. Waleffe, Spectrum of the Jacobi tau approximation for the second derivative operator, SIAM J. Numer. Anal. 46 (2007/08), 280-294. MR2377264 (2009a:65164) 
[5] M. Charalambides and F. Waleffe, Gegenbauer tau methods with and without spurious eigenvalues, SIAM J. Numer. Anal. 47 (2008), 48-68. MR2452851 (2009g:65095)

[6] T. Craven and G. Csordas, Multiplier sequences for fields, Illinois J. Math. 21 (1977), 801817. MR0568321 (58:27921)

[7] T. Craven and G. Csordas, Complex zero decreasing sequences, Methods Appl. Anal. 2 (1995), 420-441. MR1376305 (98a:26015)

[8] T. Craven and G. Csordas, Composition theorems, multiplier sequences and complex zero decreasing sequences, Value Distribution Theory and Its Related Topics (G. Barsegian, I. Laine and C. C. Yang, eds.), Kluwer Academic Press (2004). MR2173299 (2006f:26024)

[9] T. Craven and G. Csordas, The Gauss-Lucas theorem and Jensen polynomials, Trans. Amer. Math. Soc. 278 (1983), 415-429. MR697085 (85d:30031)

[10] T. Craven and G. Csordas, The Fox-Wright functions and Laguerre multiplier sequences, J. Math. Anal. Appl. 314 (2006), 109-125. MR2183541 (2007b:30030)

[11] K. Driver and K. Jordaan, Zeros of the hypergeometric polynomial $F(-n, b ; c ; z)$, arXiv:08120708v1 [math CA] 3 Dec 2008.

[12] D. Gottlieb, The stability of pseudospectral-Chebyshev methods, Math. Comp. 36 (1981), 107-118. MR595045 (82b:65123)

[13] D. Gottlieb and L. Lustman, The spectrum of the Chebyshev collocation operator for the heat equation, SIAM J. Numer. Anal. 20 (1983), 909-921. MR714688 (85g:65107)

[14] J. Garloff and D.G. Wagner, Hadamard products of stable polynomials are stable, J. Math. Anal. Appl. 202 (1996), 797-809. MR 1408355 (97e:30010)

[15] O. Holtz, Hermite-Biehler, Routh-Hurwitz, and total positivity, Linear Algebra Appl. 372 (2003), 105-110. MR1999142 (2004f:93092)

[16] A. B. J. Kuijlaars and A. Martinez-Finkelshtein, Strong asymptotics for Jacobi polynomials with varying nonstandard parameters, J. Anal. Math. 94 (2004), 195-234. MR2124460 (2005k:33006)

[17] A. B. J. Kuijlaars, A. Martinez-Finkelshtein and R. Orive, Orthogonality of Jacobi polynomials with general parameters, Electron. Trans. Numer. Anal. 19 (2005), 1-17. MR 2149265 (2006e:33010)

[18] B. Ja. Levin, Distribution of Zeros of Entire Functions, Transl. Math. Mono. 5, Amer. Math. Soc., Providence, RI, 1964; revised ed., 1980. MR0156975 (28:217)

[19] M. Marden, Geometry of Polynomials, Math. Surveys, no. 3, Amer. Math. Soc., Providence, RI, 1966. MR0225972 (37:1562)

[20] A. Martinez-Finkelshtein and R. Orive, Riemann-Hilbert analysis of Jacobi polynomials orthogonal on a single contour, J. Approx. Theory 134 (2005), 137-170. MR 2142296 (2006e:33013)

[21] N. Obreschkoff, Verteilung und Berechnung der Nullstellen reeller Polynome, VEB Deutscher Verlag der Wissenschaften, Berlin, 1963. MR0164003 (29:1302)

[22] G. Pólya, Collected Papers, Vol. II: Location of Zeros (R. P. Boas, ed.), MIT Press, Cambridge, MA, 1974. MR0505094 (58:21342)

[23] E. Rainville, Special Functions, Chelsea, New York, 1960. MR0107725 (21:6447)

[24] Q. I. Rahman and G. Schmeisser, Analytic Theory of Polynomials, Oxford University Press, 2002. MR1954841(2004b:30015)

[25] G. Szegö, Orthogonal Polynomials, Amer. Math. Soc. Colloq. Pub., vol. XXIII (4th ed.), Amer. Math. Soc., Providence, RI, 1975. MR0372517 (51:8724)

[26] D. Wagner, Zeros of reliability polynomials and $f$-vectors of matroids, Combin. Probab. Comput. 9 (2000), 167-190. MR1762787(2001f:05090)

[27] F. Waleffe, Homotopy of exact coherent structures in plane shear flows, Phys. Fluids 15 (2003), 1517-1534. MR1977897 (2004c:76045)

Mathematics, Physics, and Science Group, Frederick University, P.O. Box 24729, 1303 Nicosia, Cyprus

E-mail address: bus.chm@fit.ac.cy

Department of Mathematics, University of Hawail, Honolulu, Hawail 96822

E-mail address: george@math.hawaii.edu 